\title{
THE SYMBOLIC TREATMENT OF DIFFERENTIAL GEOMETRY*
}

\section{ARTHUR WHIPPLE SMITH}

In this paper certain theorems of differential geometry have been discussed and proved by means of a notation similar to that used in the theory of invariants. The notation was first used by Dr. H. MAschke in a paper entitled $A$ New Method of Determining the Differential Parameters and Invariants of Quadratic Differential Quantics, $\dagger$ and later, in a second paper, $A$ Symbolic Treatment of the Theory of Invariants of Quadratic Differential Quantics of $n$ Variables. $\ddagger$ The use of this notation permits the proving of theorems without any assumptions as to the character of the parameter lines by which the surface is represented and has the advantage of showing all invariant expressions in a form which is at once recognizable as invariant. No attempt has been made to outline general methods for the use of the symbols. Familiarity with the two papers of Dr. Maschke will be an aid in the acquiring of the small amount. of general method used in this paper.

In this paper the notation is first defined and certain identities are given which are easily derived from the notations. The symbolic representation of surface curves and the radius of curvature leads to the derivation of the equation of the lines of curvature and EulER's formula. Conjugate and asynptotic lines are defined and their equations obtained. Geodesics are defined frum the symbolic form for geodesic curvature and geodesic torsion is computed by means of a symbolic form for the direction cosines of the binormal.

The application of the symbols to the determination of a surface from the coefficients of the first and second fundamental forms is complete with no restrictions as to the functions chosen. The cubic form whose vanishing denotes a contact of the third order with the osculating circle is derived symbolically, as is also the quadratic form $d^{2} r^{-1} / d s^{2}$. The development of this form introduces symbols for magnitudes of order higher than two, and these symbols are used for obtaining a symbolic equation for the lines of curvature at an umbilic. A very brief treatment of the characteristic function and its equation serves

* Presented to the Society February 25, 1905. Received for publication January 15, 1905.

†Transactions of the American Mathematical Society, vol. 1 (1900), p. 197. In the sequel this is referred to as MASCHKE I.

$\ddagger$ Ibid., vol. 4 (1903), p. 445. 
to show their extreme simplicity of form under the symbolic notation. The application of symbols to the equations of the rectilinear congruence is shown to require but a slight modification of the notation already used.

\section{§1. Fundamental identities.}

This method of treatment depends primarily upon the symbolic representation of all binary quadratic differential forms as perfect squares. For this purpose we introduce symbols as follows. Assume

$$
\Sigma a_{i k} d u_{i} d u_{k}=\left(f_{1} d u_{2}+f_{1} d u_{2}\right)^{2}
$$

The symbol $f$ has actual meaning only in certain combinations, the simplest being $f_{i} f_{k}=a_{i k}=a_{k i}$. The reciprocal of $\sqrt{a_{11} a_{23}-a_{12}^{2}}$ is denoted by $\beta$. The notation $\{U V\}$ stands for $U_{1} V_{2}-U_{2} V_{1}$, where the subscripts 1 and 2 represent differentiation with respect to $u_{1}$ and $u_{2}$ respectively. The product $\beta\{U V\}$ is denoted by $(U V)$. The following theorem is fundamental : *

If $U$ and $V$ are invariants of a quadratic form, then is also $(U V)$ invariant.

The following rules are the basis for all symbolic computations:

1) In every symbolic expression of an invariant equivalent symbols may be interchanged without changing the actual value of the invariant.

2) If the symbolic expression of an invariant is changed only in sign by the interchange of two equivalent symbols, then the invariant is zero.

The more general of the formulas used are here collected for reference. The proof of those which are based directly on definitions is omitted. Those formulas which are marked * are true also for the symbol \{\} . The quantities $a, b, c, \cdots, U, V, W$ are any functions of $u_{1}$ and $u_{2}$. The symbols $f, \phi, \psi$, etc., refer to the form $E d u_{1}^{2}+2 F d u_{1} d u_{2}+G d u_{2}^{2} ; \beta$ is then the reciprocal of $\sqrt{E G-F^{2}}$. The symbols $F, \Phi, \Psi$, etc., refer to the form

$$
D d u_{1}^{2}+2 D^{\prime} d u_{1} d u_{2}+D^{\prime \prime} d u_{2}^{2} \text {. }
$$

In other words $f_{i} f_{k}=\Sigma x_{i} x_{k}, F_{i} F_{k}=-\Sigma X_{i} x_{k}, \dagger$ a sign $\Sigma$ here and elsewhere in this paper denoting summation cyclically with respect to $x, y, z$.

$$
\begin{gathered}
(a b)=-(b a), \\
(a, b c)=b(a c)+c(a b), \\
b(a b)=\frac{1}{2}\left(a b^{2}\right), \\
(a b)(c d)+(a c)(d b)+(a d)(b c)=0,
\end{gathered}
$$

- Mascher I, p. 199.

† BiANCHI-LUKAT, Differentialgeonetrie (1899), §46. In the sequel this reference is given as Bianchi. 
(5)*

$$
(a,(b c))+(b,(c a))+(c,(a b))=0,
$$

(6)* $\beta W_{2}\left(U V_{1}\right)-\beta W_{1}\left(U V_{2}\right)=\beta U_{2}\left(W V_{1}\right)-\beta U_{1}\left(W V_{2}\right)=\overline{W U V}=\overline{U W V}$;

the last equation defines the symbol $\overline{W U V}$;

$$
\begin{gathered}
(a b)_{\lambda}=\left(a_{\lambda} b\right)+\left(a b_{\lambda}\right)+\{a b\} \beta_{\lambda}, \\
(f \phi)^{2}=2, \\
(f \phi)(f U)(\phi V)=\frac{1}{2}(f \phi)^{2}(U V)=(U V), \\
(f \phi)(f,(\phi W))=0, \\
(f U)(\phi V)[(f U)(\phi V)-(f V)(\phi U)]=(U V)^{2} ; *
\end{gathered}
$$

the three formulas (9)-(11) hold also for any set of equivalent symbols, with the condition that there be also on the left such a factor that a relation similar to (8) shall exist ;

$$
f_{i} \phi_{i}(f \phi)=0, \quad \beta f_{1} \phi_{2}(f \phi)=-\beta f_{2} \phi_{1}(f \phi)=1,
$$

(13) $X=(y z)$, etc., where $X, Y, Z$ are the direction cosines of the normal to the surface,

$$
\begin{aligned}
& x_{i k}=F_{i} F_{k} X-(f \phi)(\phi x) f_{i k}, \text { etc., } \\
& X_{i}=\left(f F^{\prime}\right)(x f) F_{i} \text {, etc., } \\
& \text { (16) } \quad F_{i} \Phi_{i}(F \Phi)=0, \quad \beta F_{1} \Phi_{2}(F \Phi)=-\beta F_{2} \Phi_{1}(F \Phi)=\frac{1}{2}(F \Phi)^{2} \text {, } \\
& \Sigma(x U)(x V)=(f U)(f V), \\
& (f \phi)(\phi W)\left[\beta U_{2}\left(f_{1} V\right)-\beta U_{1}\left(f_{2} V\right)\right]=\beta W_{2}\left(U V_{1}\right)-\beta W_{1}\left(U V_{2}\right) \\
& -(f \phi)(\phi W)(U,(f V))+(\beta U)\{W V\} ;
\end{aligned}
$$

this formula, which also holds for the \{\} signs if the last term and the factors $\beta$ be omitted, can also be written

$$
-(f \phi)(\phi W) \overline{U V f}=\overline{W U V}-(f \phi)(\phi W)(U,(f V))+(\beta U)\{W V\},
$$

$$
\begin{gathered}
\Sigma(x W)((x V), U)=-(f W)(U,(f V)), \\
\Sigma X((x V), U)=(F U)(F V), \\
\left(w w_{k}\right)=-(f \phi)[\overline{w w f}+(w \beta)\{f w\}] \phi_{k},
\end{gathered}
$$

where $w_{k}$ is defined by $d u_{1}=\beta w_{2} d s, d u_{2}=-\beta w_{1} d s$,

$$
(f \phi)(\phi W)\left(F,\left(f F^{\prime}\right)\right)=0 \text {, identically in } W_{k} \text {. }
$$

\footnotetext{
* MAschKe I, p. 201.
} 
Proofs of formulas.

The following suggestions will aid in the proving of formulas. In general a parenthesis symbol is to be changed first to the symbol $B\{\}$. This should be now expanded so as to bring out the coefficients of those factors which have only the single differentiation, [e. g., formula (19)]. These coefficients will be of one of two kinds. If they involve no second differentiation of the $x, X$, etc., then formulas (1) $-(13),(16),(17)$ are usually sufficient. If such second differentiations do occur, the use of (7), (14) and (15) will reduce the case to one of the first kind.

(9) By rule (1),

$$
\begin{aligned}
(f \phi)(f U)(\phi V) & =-(f \phi)(\phi U)(f V) \\
& =\frac{1}{2}(f \phi)[(f U)(\phi V)-(f V)(\phi U)] \\
& =\frac{1}{2}(f \phi)^{2}(U V) \text { by }(4) \\
& =(U V) \text { by }(8) .
\end{aligned}
$$

(13), (14), (15). These results in Christoffel symbols as given by Branchi* are

$$
\begin{aligned}
X \sqrt{E G-F^{2}} & =\frac{\partial y}{\partial u_{1}} \frac{\partial z}{\partial u_{2}}-\frac{\partial y}{\partial u_{2}} \frac{\partial z}{\partial u_{1}}, \text { etc., } \\
\frac{\partial^{2} x}{\partial u_{1} \partial u_{2}} & =\left\{\begin{array}{c}
1 \\
1
\end{array}\right\} \frac{\partial x}{\partial u_{1}}+\left\{\begin{array}{c}
1 \\
2
\end{array}\right\} \frac{\partial x}{\partial u_{2}}+D^{\prime} X, \text { etc., } \\
\frac{\partial X}{\partial u_{1}} & =\frac{F D^{\prime}-G D}{E G-F^{2}} \frac{\partial x}{\partial u_{1}}+\frac{F D-E D^{\prime}}{E G-F^{2}} \frac{\partial x}{\partial u_{2}}, \text { etc. }
\end{aligned}
$$

From the definition of the Christoffel symbols follow

$$
\left\{\begin{array}{c}
i k \\
1
\end{array}\right\}=(f \phi) \phi_{2} f_{i k}, \quad\left\{\begin{array}{cc}
i k \\
2
\end{array}\right\}=-(f \phi) \phi_{1} f_{i k} \cdot \dot{\dagger}
$$

These notations lead to formulas (13)-(15) at once.

(18). Commence this proof by expanding $(f \phi)(\phi W)(U,(f V))$ :

$$
\begin{aligned}
& \Sigma(x W)((x V), U)=(\beta U) \Sigma(x W)\{x V\} \\
& +\beta^{2} U_{2} \Sigma(x W)\{x V\}_{1}-\beta^{2} U_{1} \Sigma(x U)\{x V\}_{2} .
\end{aligned}
$$

By (17), $\quad \Sigma(x W)\{x V\}=(f W)\{f V\}$.

By (7), $\Sigma(x W)\{x V\}_{i}=\Sigma(x W)\left\{x_{i} V\right\}+\Sigma(x W)\left\{x V_{i}\right\}$.

* BIANCHI, 88 46-47.

† BraNchi, \& 24. 
By (14),

$$
\Sigma(x W)\left\{x_{i} V\right\}=F_{i}\{F V\} \Sigma X(x W)-(f \phi)\left\{f_{i} V\right\} \Sigma(x W)(\phi x) .
$$

Since $\Sigma X x_{i}=0$ (from 13), this reduces by the aid of (17) to

$$
-(f \phi)(\psi W)(\phi \psi)\left\{f_{\mathbf{i}} V\right\},
$$

or, by change of notation, to $(f \phi)(f W)(\psi \phi)\left\{\psi_{i} V\right\}$. The term $\overline{U f} \bar{V}$ is replaced by $\overline{f U V}$ by (6). To the coefficient of $\left(f W^{\prime}\right)$ in the whole expression apply (18), using $f$ in place of $W$ and $\psi$ in in place of $f$. To this result apply (9).

\section{§ 2. Curves on a surface.}

If $U\left(u_{1} u_{2}\right)=0$ is a curve on the surface given by the equations $x=x\left(u_{1}, u_{2}\right), y=y\left(u_{1}, u_{2}\right), z=z\left(u_{1}, u_{2}\right)$, then its differential equation is $U_{1} d u_{1}+U_{2} d u_{2}=0$ and along this curve $d u_{1} / d s$ and $d u_{2} / d s$ may be replaced by $\beta_{p} U_{2}$ and $-\beta p U_{1}$ respectively, where $p$ is a factor of proportionality. The direction cosines of the tangent to any curve are $d x / d s, d y / d s, d z / d s$ or, as they may be written, $x_{1} d u_{1} / d s+x_{2} d u_{2} / d s$, etc., and in these new notations the direction cosines of $U=$ const. will be $p(x U), p(y U), p(z U)$. Since the sum of the squares of these cosines is unity the value of $p$ is easily found to be the reciprocal of $\sqrt{(f U)^{2}}=\sqrt{\Delta_{1}} U_{\text {.* }}^{*}$

Consider now two curves $U=$ const. and $V=$ const., the factors of proportionality being $p$ and $q$ respectively. Let $\omega$ be the angle between them. Then $\cos \omega=p q \Sigma(x U)(x V)$, whence is obtained $\cos \omega=p q(f U)(f V)$. From this follows $\sin ^{2} \omega=1-p^{2} q^{2}(f U)(f V)(\phi U)(\phi V)$. Consider now as one factor $p^{2} q^{2}(f U)(\phi V)$ and to the other factor apply formula (4). The result is $\sin \omega=p q(U V)$. The angle $\omega$ is defined with the condition $0 \leqq \omega<\pi$. Thus $\sin \omega$ is positive (or 0 ) and $p$ and $q$ have the same or different signs according as $(U V)$ is positive or negative. The differential invariant $\nabla(U V)$ has been found to be $(f U)(f V)$, hence the theorem :

If $U$ and $V$ are to be orthogonal curves, it is necessary and sufficient that $\nabla(U V)=0$.

If $U$ and $V$ are the parameter lines, then this condition reduces to $f_{1} f_{2}=F=0$. As a further consequence, if $V$ be the integral of the orthogonal trajectories of $U$, then their differential equation is $(f U)(f V)=0$ and from this it follows that $V_{i}=m(f U) f_{i}^{\prime}$ and by forming the expression $(\phi V)^{2}$ the factor $m$ is found to be $p / q$.

* M.AsChKE, I, p. 200 ; Bianchi, $z 23$ :

$$
\begin{gathered}
\triangle_{1} U=\beta^{2}\{\mid U\}^{2}=(f U)^{2}, \quad \nabla(U V)=\beta^{2}\{f U\}\{f V\}=(f U)(f V), \\
\triangle_{2} U=\beta\{f, \beta\{f U\}\}=(f,(f U)) .
\end{gathered}
$$


Along any curve $W=$ const. define $w_{1}$ and $w_{2}$ by the relations $d u_{1}=\beta w_{2}$ and $d u_{2}=-\beta w_{1}$. Then on this curve $d s^{2}=(f w)^{2}$. It is desirable to have a similar expression for the corresponding arc $d \sigma$ on the Gaussian sphere. By definition $d \sigma^{2}=\Sigma d X^{2}=\Sigma(X w)^{2}$. By formulas (15) and (17) this becomes $d \sigma^{2}=\left(f F^{\prime}\right)(\phi \Phi)(\phi \psi)(f \psi)(F w)(\Phi w)$. To this apply formula (9) and let $f^{\prime}, \phi^{\prime}$, etc., be symbols of $d \sigma$. Thus $d \sigma^{2}=(f F)(f \Phi)(F w)(\Phi w)=\left(f^{\prime} w\right)^{2}$ and from this identity follows $f_{i}^{\prime}=(f F) F_{i}$.

Let $U$ and $V$ form an isothermal system and also be taken as the parameter lines, then from the definition of the system must $\triangle_{1} U=\triangle_{1} V$ and $\nabla(U V)=0$.* The second of these conditions may be written $V_{i}=m(f U) f_{i}$ and the first is $(f U)^{2}=(f V)^{2}$. This is equivalent to $p= \pm q$ and therefore $m= \pm 1$. Consider naw

$$
\Delta_{2} V=(f,(f V))= \pm(f,(f \phi)(\phi U))= \pm(f \phi)(f,(\phi U)) \pm(\phi U)(f,(f \phi)) .
$$

The first term is zero by formula (10). The second term is seen to be zero by use of rule (1) and formulas (4), (3), and (8). Thus it is necessary that $\Delta_{2} V=0$. Suppose now that $U$ satisfies $\Delta_{2} U=0$ and define $V$ by the condition $V_{i}=(f U) f_{i}$. This may be done, for $\Delta_{2} U=0$ is the condition that $V_{i}$ be an exact differential. Forming $\Delta_{2} V$ from this definition of $V_{i}$ it follows as above that $\Delta_{2} V=0$. Form now $(\phi V) \phi_{i}=(f U)(\phi f) \phi_{i}$ and consider the coefficients of $U_{1}$ and $U_{2}$ for the cases $i=1,2$. It is seen that $U_{i}=-(f V), f_{i}$ and from these relations between $U$ and $V$ follow, by rule 2,

and

$$
\nabla(U V)=(f U)(f V)=(f U)(f \phi)(\phi U)=0
$$

$$
\Delta_{1} V=(f V)^{2}=(f \phi)(f \psi)(\phi U)(\psi U)=(f U)^{2}=\Delta_{1} U
$$

The function $V_{i}=(f U) f_{i}$ is called the conjugate solution to $U$. Hence the theorem :

In order that $U$ and $\boldsymbol{V}$ form an isothermal system it is a sufficient condition that $\Delta_{2} U=0$ and $V$ be the solution conjugate to $U$.

\section{§ 3. Lines of curvature.}

The radius of curvature of curves on a surface may be found as follows. From the Frenet formulas $\cos \theta / \rho=\Sigma X d \cos \alpha / d s$, where $\rho$ is the radius of curvature of the curve considered, $\alpha, \beta, \gamma$, are the direction angles of its tangent, and $\theta$ is the angle between the positive direction of the principal normal and the normal to the surface. Consider in this way the curve $U=$ const. Then $\cos \alpha=p\left(x L^{r}\right)$, etc. Therefore

$$
\Sigma X \frac{d \cos \alpha}{d s}=p^{2} \Sigma X((x U), U)-p(p U) \Sigma X(x U) .
$$

*.BIANCHI, \& 36. 
By (13) the coefficients of $U_{1}$ and $U_{2}$ in the expression $\Sigma X(x U)$ are of the form $\Sigma(y z) x_{j}$ which is identically zero. From formula $(20), \Sigma X((x U), U)=(F U)^{2}$. Whence it follows that $\cos \theta / \rho=p^{2}(F U)^{2}$. The curvature of a normal section having the same direction as $U$ is found by letting $\theta=0$ or $\pi$. If $R$ be the radius of curvature in that case, and if its sign be so chosen that $R$ is positive when the center of curvature is on the positive side of the tangential plane, then $1 / R=-p^{2}(F U)^{2}$ and $\rho=-R \cos \theta$. This is Meusnier's theorem.*

Lines of curvature are defined as those curves along which the radius of curvature is a maximum or a minimum when considered as dependent on the direction in which the curve passes through a point. For any one value of $\theta$ consider all the curves which pass through any one point. Then, at this point, from the relation $\rho=-R \cos \theta, \rho$ is a maximum or a minimum according as $R$ is a maximum or a minimum. It remains then to find those curves along which $R$ is a maximum or minimum. The parameter of the normal section is $d u_{1} / d u_{2}$. Put $w=U_{2} / U_{1}$, then (using $\rho$ for $R$ ),

Therefore

$$
\rho=-\frac{(f U)^{2}}{(F U)^{2}}=-\frac{\left(f_{1} w-f_{2}\right)^{2}}{\left(F_{1}^{\prime} w-F_{2}\right)^{2}} \text {. }
$$

$$
-\frac{d \rho}{d w}=\frac{2\left(F_{1} w-F_{2}\right)^{2}\left(f_{1} w-f_{2}\right) f_{1}-2\left(f_{1} w-f_{2}\right)^{2}\left(F_{1}^{\prime} w-F_{2}\right) F_{1}}{\left(F_{1} w-F_{2}\right)^{4}} .
$$

By removing $w$ and simplying the numerator the condition for a maximum or a minimum becomes $\left(f F^{\prime}\right)(f U)(F U)=0$ and this is the differential equation of the lines of curvature. This equation may also be written in the form $U_{i}=h(f F)(F U) f_{i}$ and $h$ may be determined as a function of $U$ by forming $(\phi U)^{2}$.

Let $U$ be a line of curvature and $V$ be orthogonal to $U$. Then $U_{i}=n(f V) f_{i}$. If this be substituted in the equation for $U$ the equation $\left(f F^{\prime}\right)(f \phi)(F \psi)(\phi V)(\psi V)=0$ is obtained. This reduces to

$$
(f F)(f V)(F V)=0,
$$

whence it appears that the lines of curvature form an orthogonal system.

It has been found that $X_{i}=(f F)(x f) F_{i}$. Let now $U$ be a line of curvature so that $(f F)(f U)(F U)=0$. Then

$$
(\phi U)^{2}(X U)=(f F)(x f)(F U)(\phi U)^{2} .
$$

Apply (4) to $\left(f F^{\prime}\right)(\phi U)$ and then (9) to $(x f)(\phi U)(f \phi)$. These reductions give $(X U)=-p^{2}(F U)^{2}(x U)$. But since along $U$ the expressions $(X U)$ and $(x U)$ are proportional to $d X$ and $d x$ respectively, the following theorem is proved :

* BIANCHI, §53. 
Along a line of curvature the ratio $d x: d X$ equals the radius of curvature.

\section{§4. Conjugate Lines.*}

We define as conjugate lines those whose directions satisfy the equation $\tan \theta_{1} \tan \theta_{2}=-r_{1} / r_{2}$, where $\theta_{1}$ and $\theta_{2}$ are the angles which the two curves make with the line of curvature $V$, and $r_{1}$ and $r_{2}$ are the values of $\rho$ along $U$ and $V$ respectively. Let $W$ and $T$ be conjugate curves. From the expressions previously found for sine and cosine a form for the tangent function may be derived, and from this the differential equation satisfied by $W$ and $T$ is

$$
\frac{(W V)(T V)}{(f T)(f V)(\phi W)(\phi V)}=\frac{-(f U)^{2}(F V)^{2}}{(. f V)^{2}(\overline{F U})^{2}}
$$

To the expressions $(f U)(F V)$ and $(f V)(F U)$ apply formula (4) and note that $(f U)(f V)=0$. If the equation be now cleared of fractions, the expression $(f F)(f U)(F V)(\phi W)(\phi V)(\psi T)(\psi V)$ appears. Apply (4) to $(f U)(\phi V),(W V)(F U)$, and $(T V)\left(f F^{\prime}\right)$, remembering that $U$ and $V$ are lines of curvature. If now the right member be reduced by means of (9), the resulting equation of the conjugate lines is $(F W)(F T)=0$. From this it follows that if $U$ and $V$ be conjugate lines, then $U_{i}=k(F V) F_{i}$, and if this value of $U_{i}$ be substituted in $\left(f F^{r}\right)(f U)(F U)=0$, it is seen that $V$ is a line of curvature. It follows then that the lines of curvature are conjugate.

Also if two curves are conjugate and orthogonal they are lines of curvature. For, in $U_{i}=k(F V) F_{i}$ put $V_{i}=n(f U) f_{i}$ and then form $(U U)$ which is itentically zero. The result is $\left(f F^{\prime}\right)(f U)(F U)=0$. Thus the conditions of conjugacy and orthogonality might be taken as the definition of lines of curvature.

Asymptotic lines are defined as those along which the two conjugate directions coincide. Their equation is then $\left(F C^{\top}\right)^{2}=0 . \dagger$

\section{§5. Principal Radir. $\ddagger$}

The quadratic for the principal radii may be set up as follows:

$$
-\left(r_{1}+r_{2}\right)=\frac{(F V)^{2}(f U)^{2}+(F U)^{2}(f V)^{2}}{(F U)^{2}(\Phi V)^{2}} .
$$

Apply (4) to $(F V)(f U)$ and to $(F U)(f V)$, using at the same time the conditions of orthogonality and conjugacy. The numerator becomes then $(U V)^{2}\left(f^{\prime}\right)^{2}$. To the denominator apply (4) and (9). The result is $\frac{1}{2}(U V)^{2}(F \Phi)^{2}$. Also $r_{1} r_{2}=(f U)^{2}(\phi V)^{2} /(F U)^{2}(\Phi V)^{2}$ and by a similar process this is reduced to $2 /(F \Phi)^{2}$. The desired quadratic is then

* Bianchi, \& 8 54, 56.

† BIANCHI, \& 57.

$\ddagger$ BIANCHI, 852. 


$$
(F \Phi)^{2} r^{2}+2(f F)^{2} r+2=0 .
$$

Let the total curvature $1 / r_{1} r_{2}$ be denoted by $K$ and the mean curvature $1 / r_{1}+1 / r_{2}$ by $-H$, then it follows that $2 K=(F \Phi)^{2}$ and $H=\left(f F^{2}\right)^{2}$, while the equation becomes $K r^{2}+H r+1=0$.

Euler's formula. * Let $W=$ const. be any curve, $U, V$ the lines of curvature, and $\theta$ the angle which $W$ makes with $V(0 \leqq \theta<\pi)$. Then

$$
\frac{1}{r_{1}}=-p^{2}(F U)^{2} \quad \text { and } \quad \frac{1}{r_{2}}=-q^{2}(F V)^{2} .
$$

By the condition of orthogonality $U$ may be eliminated from the expression for $r_{1}$, the result being

Also

$$
-\frac{1}{r_{\mathrm{t}}}=\frac{\left(f F^{\prime}\right)(\phi F)(f V)(\phi V)}{(f \phi)(f \psi)(\phi V)(\psi V)}=q^{2}\left(f F^{\prime}\right)(\phi F)(f V)(\phi V)
$$

$$
\sin ^{2} \theta=q^{2} \frac{(W V)^{2}}{(f W)^{2}}, \quad \cos ^{2} \theta=q^{2} \frac{(f W)(\phi W)(f V)(\phi V)}{(f W)^{2}} .
$$

Form now in symbols the expression $-\left[\cos ^{2} \theta / r_{2}+\sin ^{2} \theta / r_{1}\right]$, reducing it to a fractional form. In the numerator apply formula (4) to the expressions $(W V)(\phi F)$ and $(F V)(f W)$ and collect the coefficients of $(f V)(F W)$ and $(W V)(F V)$. By an interchange of $f$ and $\phi$, the first of these becomes $-[(\phi W)(F V)+(W V)(\phi F)](f V)(\phi V)$, which is to be reduced again hy means of (4). The complete term involving $(W V)\left(F^{\prime} V\right)$ contains the factor $(f F)(f V)(F V)$ and is therefore zero. This result in its simplified form is Euler's formula, viz :

$$
\frac{1}{r}=\frac{\cos ^{2} \theta}{r_{2}}+\frac{\sin ^{2} \theta}{r_{1}}
$$

In the process of finding the sum $-\left(r_{1}+r_{2}\right)$ only the condition of conjugacy needs to be used. Hence the theorem :

The sum of the radii of curvature of two conjugate normal sections is constant and is $-H / K$.

If a similar process be applied to the sum of the curvatures of two orthogonal normal sections, then follows the theorem :

The sum of the curvatures of two orthogonal normal sections is constant and is $-H$.

\section{§ 6. Geodesic curvature. $\dagger$}

At any point of a curve $C$ on a surface consider the projection of $C$ on the tangent plane. The ordinary curvature of this new curve is called the geodesic

* Bianchi, \& 54.

† BIANCHI, \& 75. 
curvature of the curve $C$. Its computation is as follows. Let $U$ and $V$ be any two orthogonal curves and let it be required to find the geodesic curvature of $U$. Let $1 / \rho$ and $1 / \rho_{g}$ be the ordinary and the geodesic curvatures respectively. Let $\theta$ be the angle between the principal normal to $U$ and the principal normal to the curve on the tangent plane. Then $1 / \rho_{g}=\cos \theta / \rho .^{*}$ From the Frenet formulas we have $\cos \xi / \rho=d \cos \alpha / d s$ etc., where $\xi, \eta, \zeta$ and $\alpha, \beta, \gamma$ are the direction angles of the principal normal and the tangent respectively. Along $U$ we have $\cos \alpha=p(x U)$, etc., and therefore

$$
\frac{\cos \xi}{\rho}=n(p(x U), U)=p^{2}((x U), U)+p(p U)(x U) .
$$

Since the principal normal to a curve lies in its osculating plane, that of the projected curve is then the tangent to the curve $V$. Its direction cosines are therefore $q(x V)$, etc. Whence it follows that

$$
\frac{1}{\rho_{g}}=p^{2} q \Sigma((x U), U)(x V)+p q(p U) \Sigma(x U)(x V) .
$$

Since $U$ and $V$ are orthogonal, $\Sigma(x U)(x V)=(f U)(f V)=0$ and $V_{i}=m(f U) f_{i}$, whence $1 / \rho_{g}=p^{2} q m(\chi U) \Sigma(x \chi)((x U), U)$. If in formula (19) $\chi$ is substituted for $W$ and $U$ for $V$ it follows that

$$
\Sigma(x \chi)((x U), U)=(f \phi)(\phi \psi)(\psi \chi)(U,(f U))=(\chi f)(U,(f U)) .
$$

The relation $m q=p$ gives as a final result

$$
\frac{1}{\rho_{g}}=-p^{3}(f \phi)(\phi U)(U,(f U)) .
$$

The invariant character of this expression is evident from its form.

Geodesic lines are now defined as those curves along which the geodesic curvature is zero. The symbolic form of the equation of the geodesics is then

$$
(f \phi)(\phi U)(U,(f U))=0 .
$$

\section{§ 7. The orthogonal trajectories of geodesics.}

Let $U$ be such a curve that its orthogonal trajectories may be given by $V_{i}=p(f U) f_{i}$. The usual form is $V_{i}=m(f U) f_{i}$ and since $m q=p$, it must be that in this case $q=1$, i. e., $\Delta_{1} V=1$. The necessary condition that $U_{i}$ be an exact differential is $(f, p(f U))=0$ or $p(f,(f U))+(f p)(f U)=0$. Divide the members of this equation by $p^{3}$, and write the expression $p^{-3}(f p)$ as $-\frac{1}{2}\left(f, 1 / p^{2}\right)$. Replace $p^{-2}$ by $(\phi U)^{2}$ and apply (4) to the expression $(\phi U)(f,(f U))$. The resulting equation is $(f \phi)(\phi U)(U,(f U))=0$. Hence the theorem :

\footnotetext{
* Meusnier's theorem, \& 3.
} 
If the orthogonal trajectories of a curve are given by $V_{i}=p(f U) f_{i}$ then the curve is a geodesic and $\Delta_{1} V=1$.

If $\alpha$ be the constant of integration in $V$, then

$$
\frac{\partial \triangle_{1} V}{\partial \alpha}=2(f V) \frac{\partial(f V)}{\partial \alpha}=2(f V)\left(f, \frac{\partial V}{\partial \alpha}\right)=0 .
$$

Therefore $\nabla(V, \partial V / \partial \alpha)=0$ and the curves $\partial V / \partial \alpha=$ const. are the orthogonal trajectories of the curves $V=$ const.

From this follows the theorem:

The integral equation of the geodesics in terms of their orthogonal trajectories $V$ is $\partial V / \partial \alpha=0$.

\section{§ 8. The cosines OF THE BINORMaL.}

The direction cosines of the binormal of a curve are proportional to the differences $\left(y^{\prime} z^{\prime \prime}-z^{\prime} y^{\prime \prime}\right)$, etc., where the accents denote differentiation with respect to s. Along any curve $W$ let

$$
d u_{1}=\beta w_{2} d s=\beta t W_{2} d s \quad \text { and } \quad d u_{2}=-\beta w_{1} d s=-\beta t W_{1} d s,
$$

these relations being the definitions of $w_{i}$ and $t^{2}=(f W)^{2}$.

Then $y^{\prime}=(y w), y^{\prime \prime}=((y w), w)$ and therefore

$$
\begin{aligned}
y^{\prime} z^{\prime \prime}-z^{\prime} y^{\prime \prime} & =(y w)((z w), w)-(z w)((y w), w) \\
& =\beta w_{2}\left[(y w)\left(z_{1} w\right)-(z w)\left(y_{1} w\right)\right]-\beta w_{1}\left[(y w)\left(z_{2} w\right)-(z w)\left(y_{2} w\right)\right] \\
& +\beta w_{2}\left[(y w)\left(z w_{1}\right)-(z w)\left(y w w_{1}\right)\right]-\beta w_{1}\left[(y w)\left(z w_{2}\right)-(z w)\left(y w w_{2}\right)\right] .
\end{aligned}
$$

Apply (4) to the third and fourth terms obtaining $\beta w_{2}(y z)\left(w w_{1}\right)$. and $-\beta w_{1}(y z)\left(w w_{2}\right)$ respectively. From the identity $(y w)\left(z_{i} w\right)=\beta^{2}\{y w\}\left\{z_{i} w\right\}$ compute the differences $(y w)\left(z_{i} w\right)-(z w)\left(y_{i} w\right)$. These contain terms $y_{j} z_{i k}-z_{j} y_{i k}$ which, by the elimination of $z_{i k}, y_{i k}$, become

$$
F_{i} F_{k}\left[y_{j} Z-z_{j} Y\right]-(f \phi)\left[y_{j}(\phi x)-z_{j}(\phi y)\right] f_{i k} .
$$

If $Z$ and $Y$ be expressed in terms of $x, y, z$ then

$$
y_{j} Z-z_{j} Y=-(f x) f_{j} . \quad \text { Also } \quad y_{j}(\phi z)-z_{j}(\phi y)=(y z) \phi_{j} .
$$

From these results follows

Consequently

$$
y_{j} z_{i k}-z_{j} y_{i k}=-F_{i} F_{k}(f x) f_{j}-(f \phi)(y z) \phi_{j} f_{i k} .
$$

$$
(y w)\left(z_{i} w\right)-(z w)\left(y_{i} w\right)=-(f x)(f w)(F w) F_{i}-(f \phi)(\phi w)\left(f_{i} w\right)(y z) .
$$


If this be substituted in the original equation, the result is

$$
\begin{array}{r}
y^{\prime} z^{\prime \prime}-z^{\prime} y^{\prime \prime}=(y z)\left[\beta w_{2}\left(w w_{1}\right)-\beta w_{1}\left(w w_{2}\right)-(f \phi)(\phi w)\left\{\beta w_{3}\left(f_{1} w\right)-\beta w_{1}\left(f_{2} w\right)\right\}\right] \\
-(f x)(f w)(F w)^{2} .
\end{array}
$$

In formula (18) put $U=V=W=w$ and apply it to the coefficient of $(y z)$. If $t^{3}(f \phi)(\phi W)(W,(f W))$ be denoted by $G$ and if the notation $W_{i}$ be used instead of $w_{i}$, the desired proportion takes the form

$$
\begin{array}{r}
\cos \lambda: \cos \mu: \cos \nu=G(y z)-t^{3}(f x)(f W)(F W)^{2}: G(z x)-t^{3}(f y)(f W)(F W)^{2} \\
: G(x y)-t^{3}(f z)(f W)(F W)^{2} .
\end{array}
$$

From this are easily derived expressions for the cosines of the principal normal of a geodesic. For if $\xi, \eta, \zeta$ be the direction angles, then

$$
\cos \xi=\cos \mu \cos \gamma-\cos \nu \cos \beta, \text { etc. }
$$

Along a geodesic $G$ is zero and therefore

$$
\cos \lambda: \cos \mu: \cos \nu=(f x)(f W):(f y)(f W):(f z)(f W) \text {. }
$$

Also $\cos \alpha: \cos \beta: \cos \gamma=(x W):(y W):(z W)$ and by substitution in the above identity it follows that

$$
\cos \xi: \cos \eta: \cos \zeta=(y z):(z x):(x y) .
$$

But since $\Sigma(x y)^{2}=1$,

$$
\cos \xi= \pm X, \quad \cos \eta= \pm Y, \quad \cos \zeta= \pm Z .
$$

For an asymptotic line $(F W)^{2}=0$ and therefore as above

$$
\cos \lambda= \pm X, \quad \cos \mu= \pm Y, \quad \cos \nu= \pm Z .
$$

From these considerations follows the theorem:

The surface normal along a geodesic line coincides with the principal normal and along an asymptotic line with the binormal.

\section{§9. Geodesic torsion.}

The Frenet expression for torsion is $1 / T=-\Sigma \cos \lambda d \cos \xi / d s$. $^{*}$ Let $U$ be a geodesic line. Then along $U, \cos \xi= \pm X$, etc., $\cos \lambda=\mp p(f x)(f U)$. The factor of proportionality is found to be $p$ by evaluating the expression $\Sigma[(f x)(f U)]^{2}$. Its sign is - or + according as $\cos \xi$ is $\pm X$, as is seen from $\cos \alpha=\cos \eta \cos \nu-\cos \zeta \cos \mu$. Also from (15),

$$
\frac{d \cos \xi}{d s}= \pm p\left(X U^{T}\right)= \pm p(f F)(x f)(F U) .
$$

* Bianchi, 884 . 
From these relations with $(9), 1 / T=-p^{2}(f F)(f U)(F U)$. If now $U$ is not a geodesic then this is by definition the geodesic torsion of $U$. In other words the geodesic torsion of a curve is the torsion of that geodesic which is tangent to it in the point considered. For a geodesic the two torsions are identical.

Suppose that $U$ and $V$ are orthogonal. The geodesic torsion of $V$ is $-q^{2}(f F)(f V)(F V)$. If in this $m(f U) f_{i}$ be substituted for $V_{i}$, the result is $p^{2}(f F)(f U)(F U)$ and hence the geodesic torsions of orthogonal curves differ only in sign.

Let $\theta$ be the angle between the surface normal and the principal normal to the curve $U$. Then

$\cos \xi=X \cos \theta+\cos \alpha \sin \theta \quad$ and $\quad \cos \lambda=-X \sin \theta+\cos \alpha \cos \theta, *$

where $\cos \alpha=q(x V)$. These values for $\cos \xi$ and $\cos \lambda$ are to be substituted in the expression for torsion and in the reductions it is to be noticed that $\Sigma X(X U)=0, \Sigma X(x V)=0, \Sigma(x V)^{2}=q^{-2}, \Sigma X((x V), U)=(F U)(F V)$, $\Sigma(x V)(X U)=-(F U)(F V), \Sigma(x V)((x V), U)=q(q U)$. These identities are proved in $\S 1$, the last being a special case of $(19)$. The resulting equation is $1 / T=-p(\theta U) X+p q(F U)(F V)$, and from this $q$ and $V$ may be removed by the condition of orthogonality. The equation then takes the form $1 / T+d \theta / d s=-p^{2}(f F)(f U)(F U)$.

This is equivalent to the theorem:

The difference between the geodesic and ordinary torsions of a curve is the derivative with respect to $s$ of the angle between the surface normal and the principal normal of the curve.

From the definition of geodesic torsion follows the theorem :

If a line of curvature is a geodesic, it is plane.

It has already been proved that along a geodesic $\theta=0$, and the above expression for $\cos \lambda$, etc., together with the conclusions of $\S 8$ show that along an asymptotic curve, $\theta=+\pi / 2$.

\section{$\$ 10$. The Codazzi formulas, $\beta$ and $\beta^{\prime}, f$ and $f^{\prime}$.}

The relation $\beta=\beta^{\prime} K$.

The function $\beta$ has been defined and is always positive, $K$ is the total curvature given symbolically in $\S 5$, and $\beta^{\prime}$ is defined for the Gaussian are as was $\beta$ for the ordinary arc except that its sign is not fixed. It has been proved that $f_{i}^{\prime}=\left(f F^{\prime}\right) F_{i}^{\prime}$. From this form the expression $\left\{f^{\prime} \phi^{\prime}\right\}^{2}$ may be reduced by the process of formula (9) to $\{f \phi\}^{2} K^{2}$, and gives the desired relation.

The symbol $f$ in terms of $f^{\prime}$ and $F$.

The consideration of $\left(f^{\prime} \Phi\right) \Phi_{i}=\left(f F^{\prime}\right)(F \Phi) \Phi_{i}$ in the light of formula (16), for the two cases $i=1,2$, leads to the equation $-K f_{i}=\left(f^{\prime} F^{\prime}\right) F_{i}$.

* Bra NCHI, 885 . 


\section{The Codazzi formulas.*}

These formulas are together equivalent to the identical vanishing of the covariant $(f \phi)(\phi F)((f F), w) \cdot \dagger$ Therefore $(f \phi)\left(\phi F^{\prime}\right)\left(f F^{\prime}\right)_{k}=0$ where $k=1,2$. The application of formula (7) gives

$$
(f \phi)(\phi F)\left(f_{k} F^{\prime}\right)+(f \phi)\left(\phi F^{\prime}\right)\left(f F_{k}\right)+(f \phi)(\phi F)\left\{f F^{\prime}\right\} \beta_{k}=0 .
$$

Rule 2 applied to the third term and (9) to the second give

$$
\left(F F_{k}\right)=(f \phi)(F \phi)\left(F f_{k}\right) \text {. }
$$

The direct derivation of these formulas is as follows. The identity $x_{i k i}-x_{i i k}=0$, when reduced by means of (14), may be written

$$
\left[F_{i} F_{k} X\right]_{i}-\left[F_{i} F_{i} X\right]_{k}=\left[(f \phi)(\phi x) f_{i k}\right]_{i}-\left[(f \phi)(\phi x) f_{i i}\right]_{k}
$$

and this is equivalent to

$$
X\left(F_{k} F_{i i}-F_{i} F_{i k}\right)-\left(F_{k} X_{i}-F_{i} X_{k}\right) F_{i}=[(f \phi)(\phi x)]_{i} f_{i k}-[(f \phi)(\phi x)]_{k} f_{i i} \text {. }
$$

Since in this identity $i \neq k$, it may be written

$$
X\left(F F_{k}\right)-(F X) F_{k}=\left(f_{k},(f \phi)(\phi x)\right) .
$$

Multiply this by $X$ and take the cyclic sum, applying (20) to $\Sigma X\left(f_{k},(\phi x)\right)$. The equation is then reduced to the usual form, viz.,

$$
\left(F F_{k}\right)=(f \phi)(F \phi)\left(F f_{k}\right) \text {. }
$$

§ 11. The differential equations Required For the Determination OF A SURFACE OF WHICH THE FIRST AND SECOND FUNDAMENTAL FORMS ARE GIVEN. $\ddagger$

Consider a set of axes consisting of the normal at any point of the surface and the tangents to two curves $U$ and $V$ which pass through the foot of the normal. No supposition is made as to the character or relative positions of $U$ and $V$. Let the direction cosines of the normal and the tangents to $U$ and $V$ be $X, Y, Z, X^{\prime}, Y^{\prime}, Z^{\prime}, X^{\prime \prime}, Y^{\prime \prime}, Z^{\prime \prime}$ respectively. Then in accordance with the results of $\S 2$,

$$
X^{\prime}=p(x U), X^{\prime \prime}=q(x V), \text { etc. }
$$

It is desired to find the values of $X_{\lambda}, X_{\lambda}^{\prime}, X_{\lambda}^{\prime \prime}$ etc., in terms of the given coefficients and the functions $U$ and $V$. By formula (7),

$$
X_{\lambda}^{\prime}=(x U) p_{\lambda}+p\left(x_{\lambda} U\right)+p\left(x U_{\lambda}\right)+p\{x U\} \beta_{\lambda} .
$$

* Branchi, 8 48.

† Proved by Dr. MAsch KE, in a course of lectures at Chicago in 1902.

‡ BIANCHI, \& 49. SCHFFFrRs, Anwendung der Differential- und Integral-rechnung auf Geometrie (1902), vol. 2, p. 310. 
From the two equations $X==p(x U)$ and $X^{\prime \prime}=q(x V)$ it follows that $x_{i}=t\left[X^{\prime} V_{i} / p-X^{\prime \prime} U_{i} / q\right]$ where $t(V U)=1$. For the evaluation of $X_{\lambda}^{\prime}$, it is to be noted that

$$
(x U)=\frac{X^{\prime}}{p}, \quad\left(x U_{\lambda}\right)=\frac{t X^{\prime}}{p}\left(V U_{\lambda}\right)-\frac{t X^{\prime \prime}}{q}\left(U U_{\lambda}\right), \quad\{x U\}=\frac{t X^{\prime}}{p}\{V U\} .
$$

By (14),

$$
\begin{aligned}
\left(x_{\lambda} U\right) & =(F U) F_{\lambda} X-(f \phi)(\phi x)\left(f_{\lambda} U\right) \\
& =(F U) F_{\lambda} X-\frac{t X^{\prime}}{p}(f \phi)(\phi V)\left(f_{\lambda} U\right)+\frac{t X^{\prime \prime}}{q}(f \phi)(\phi U)\left(f_{\lambda} U\right) .
\end{aligned}
$$

Let $m q=p$ and $n p=q$. Let $R^{\prime}, S^{\prime}, T^{\prime}, R^{\prime \prime}, S^{\prime \prime}, T^{\prime \prime}$ be the proper coefficients in the identities

$$
\begin{aligned}
& p X_{\lambda}^{\prime}=R^{\prime} X+S^{\prime} X^{\prime}+T^{\prime} X^{\prime \prime}, \\
& q X_{\lambda}^{\prime \prime}=R^{\prime \prime} X+S^{\prime \prime} X^{\prime}+T^{\prime \prime} X^{\prime \prime} .
\end{aligned}
$$

Then follow from the identities above

$$
\begin{aligned}
R^{\prime} & =p^{2}(F U) F_{\lambda}, \\
S^{\prime} & =p_{\lambda}-p t(f \phi)(\phi V)\left(f_{\lambda} U\right)+p t\left(V U_{\lambda}\right)+p t\{V U\} \beta_{\lambda}, \\
T^{\prime} & =p m t(f \phi)(\phi U)\left(f_{\lambda} U\right)-p m t\left(U U_{\lambda}\right)=p m t(f \phi)(\phi U)(f U)_{\lambda} .
\end{aligned}
$$

The coefficients $R^{\prime \prime}, S^{\prime \prime}, T^{\prime \prime}$ are obtained from $R^{\prime}, T^{\prime}, S^{\prime}$ respectively by changing $p$ to $q, m$ to $n, U$ to $V, V$ to $U$, and $t$ to $-t$. Therefore

$$
\begin{aligned}
& R^{\prime \prime}=q^{2}(F V) F_{\lambda}, \\
& S^{\prime \prime}=-q n t(f \phi)(\phi V)\left(f_{\lambda} V\right)+q n t\left(V V_{\lambda}\right)=-q n t(f \phi)(\phi V)(f V)_{\lambda}, \\
& T^{\prime \prime}=q_{\lambda}+q t(f \phi)(\phi U)\left(f_{\lambda} V\right)-q t\left(U V_{\lambda}\right)-q t\{U V\} \beta_{\lambda} .
\end{aligned}
$$

The partial derivatives of $X$ are obtained as follows : $X X_{\lambda}=-X^{\prime} X_{\lambda}^{\prime}-X^{\prime \prime} X_{\lambda}^{\prime \prime}$ and therefore follows

$p q X X_{\lambda}=-X\left[q R^{\prime} X^{\prime}+p R^{\prime \prime} X^{\prime \prime}\right]-X^{\prime 2} q S^{\prime}-X^{\prime} X^{\prime \prime}\left[q T^{\prime}+p S^{\prime \prime}\right]-X^{\prime \prime 2} p T^{\prime \prime}$.

All the derivatives are thus determined. If now the curves $U$ and $V$ are supposed to be. orthogonal, further simplifications may be made. By the relations $V_{i}=m(f U) f_{i}$ and $U_{i}=n(f V) f_{i}$ remove the $V$ from $p S^{\prime}$ and the $U$ from $q T^{\prime \prime}$. In this reduction the converse of formula (7) is used, $m t=p^{2}$, and $(f U)^{2}$ is to be replaced by $p^{-2}$. The similar operations are to be used in the other case. The results are $S^{\prime \prime}=T^{\prime \prime}=0$. Consider also the expression $q T^{\prime}+p S^{\prime \prime} . \quad$ Aside from a factor $p q t$ this may be written

$$
m(f \phi)(\phi U)\left(f_{\lambda} U\right)-n(f \phi)(\phi V)\left(f_{\lambda} V\right)-m\left(U U_{\lambda}\right)+n\left(V V_{\lambda}\right) .
$$

The condition of orthogonality together with $m n=1$ gives for this 


$$
(f V)\left(f_{\lambda} U\right)-(f U)\left(f_{\lambda} V\right)-(f V)\left(f U_{\lambda}\right)+(f U)\left(f V_{\lambda}\right) .
$$

By formula (7) remove $\left(f U_{\lambda}\right)$ and $\left(f V_{\lambda}\right)$. The result obtained is zero. Therefore for the case of orthogonal curves the desired relations are

$$
\begin{aligned}
p q X_{\lambda} & =-q R^{\prime} X^{\prime}-p R^{\prime \prime} X^{\prime \prime} \\
p X_{\lambda}^{\prime} & =R^{\prime} X+T^{\prime} X^{\prime \prime} \\
q X_{\lambda}^{\prime \prime} & =R^{\prime \prime} X+S^{\prime \prime} X^{\prime} .
\end{aligned}
$$

\section{$\S \S 12,13$. The CUBIC FORM $d r^{-1} / d s .^{*}$}

$\S 12$. In every point of a surface there is at least one normal section which has a contact of the third order with the corresponding circle of curvature. The investigation of this involves the condition $d r=0$ or $d r^{-1}=0$, where $r$ is the radius of curvature of the section. Let $W$ be the integral of the curve and put $d u_{1}=\beta w_{2} d s=\beta p W_{2} d s$, and $d u_{2}=-\beta w_{1} d s=-\beta p W_{1} d s$. The symbol $p$ is here defined by the relation $p^{-2}=(f W)^{2}$ : Expressed with these symbols $1 / r$ is $(F w)^{2}$ and therefore follows $d r^{-1} / d s=2(F w)((F w), w)$. If this expression be expanded by use of $(6)$, it may be written

$$
2(F w)[\overline{F w w}-\overline{w w F}]+2\{\beta w\}(F w)^{2} .
$$

The reduction of this to an invariant form is as follows. By (21),

$$
\begin{aligned}
\overline{F w w} & =(f \phi)(\phi F)\left[\beta w_{2}\left(f_{1} w\right)-\beta w_{1}\left(f_{2} w\right)-\{w \beta\}\{f w\}\right] \\
& =(f \phi)(\phi F)\left[\beta w_{2}\left(f_{1} w\right)-\beta w_{1}\left(f_{2} w\right)\right]+(F w)\{w \beta\},
\end{aligned}
$$

by (9). By (8),

Change $\overline{F w w}$ by means of the identity

$$
-2 \overline{w w F}=-(f \phi)^{2} \overline{w w F} .
$$

$$
(f \phi)(\phi F)\left(f_{k} w\right)=(f \phi)\left[(\phi w)\left(f_{k} F\right)+\left(\phi f_{k}\right)(F w)\right],
$$

and change $-2 \overline{w w} \bar{F}$ by means of

$$
(f \phi)^{2}\left(F_{k} w\right)=2(f \phi)(\phi w)\left(f F_{k}\right)
$$

((4) and rule 1). Then

$$
\begin{aligned}
\frac{d r^{-1}}{d s}= & 2(f \phi)(\phi w)(F w)\left[\beta w_{2}\left[\left(f_{1} F\right)+\left(f F_{i}\right)\right]-\beta w_{1}\left[\left(f_{2} F\right)+\left(f F_{2}\right)\right]\right] \\
& +2(F w)^{2}\{w \beta\}+2(f \phi)(F v)^{2}\left\lceil\beta w_{2}\left(\phi f_{1}\right)-\beta w_{1}\left(\phi f_{2}\right)\right]+2\{\beta w\}(F w)^{2} .
\end{aligned}
$$

By rule 1,

$$
2(f \phi)\left(\phi f_{k}\right)=(f \phi)\left[\left(\phi f_{k}\right)+\left(\phi_{k} f\right)\right]
$$

\footnotetext{
* Knoblauch, Theorie der krummen Flächen (1888), pp. 92 and 107.
} 
and therefore by (7)

$$
\begin{aligned}
\frac{d r^{-1}}{d s}=2( & f \phi)(\phi w)(F w)\left[\beta w_{2}(f F)_{1}-\beta w_{1}(f F)_{2}-\beta w_{2}\{f F\} \beta_{1}+\beta w_{1}\{f F\} \beta_{2}\right] \\
& +(f \phi)(F w)^{2}\left[\beta w_{2}(\phi f)_{1}-\beta w_{1}(\phi f)_{2}-\beta w_{2}\{\phi f\} \beta_{1}+\beta w_{1}\{\phi f\} \beta_{2}\right] .
\end{aligned}
$$

In the reduction of this, $2(f \phi)((\phi f), v)$ is zero by (3) and (8). Also, by (19),

$$
-2(f \phi)(\phi w)(F w)\left\{f F^{\prime}\right\}(\beta w)=-2(F w)^{2}\{\beta w\}
$$

and

therefore

$$
-(f \phi)\{\phi f\}(\beta w)=(f \phi)^{2}\{\beta w\}=2\{\beta w\} ;
$$

$$
\frac{d r^{-1}}{d s}=2(f \phi)(\phi w)(F w)((f F), w) \text {. }
$$

Introduce now the $W_{i}$, and then follows

$$
P=\frac{d r^{-1}}{d s}=2 p^{3}(f \phi)(\phi W)(F W)((f F), W) .
$$

Consider the coefficients of this form. If for the moment $W_{1}$ and $W_{2}$ be replaced by $w_{2}$ and $-w_{1}$ respectively, then $P$ may be written in the form $\Sigma P_{i j k} w_{i} w_{j} w_{k}$, where $i, j, k=1,2,3$, and $P_{i j k}=2 p^{3} \beta^{3}(f \phi)\left(f F^{\prime}\right)_{k} F_{j} \phi_{i}$, the $i, j, k$ being of course interchangeable, which may, however, be proved independently of the definition of $P_{i j k}$.

- The collected coefficients of this cubic are called fundamental magnitudes of the third order * and may now properly be expressed by symbols. Put then $P_{i j k}=\pi_{i} \pi_{j} \pi_{k}$, which defines the symbols $\pi$.

If now the $W_{i}$ be again introduced, $P=(\pi W)^{3}$, where the symbols $\pi$ are defined by $\pi_{i} \pi_{j} \pi_{k}=2 p^{3} \beta^{3}(f \phi)(f F)_{k} \phi_{j} F_{i}$.

The form $T=d^{2} r^{-1} / d s^{2}$ may be treated in like manner. The synubolic form of $T$ is obtained by differentiating $P$ and is therefore $3 p(\pi W)^{2}$ $((\pi W), W)$. The quantities $W_{i k}$ may be removed by exactly the same process as that used above; since in the process there employed, no use was made of the peculiar properties of $F$, in place of which $T$ contains $\pi$. Therefore

$$
T=3 p(\pi W)^{2}((\pi W), W)=3 p(f \phi)(\phi W)(\pi W)^{2}((f \pi), W) .
$$

The introduction of new symbols, $\tau_{i} \tau_{j} \tau_{k} \tau_{\lambda}$ for the coefficients of $T$, and in fact the continuance of this process, will depend upon the permutability of the indices. The use of such combinations as those represesenting $\pi_{i} \pi_{j} \pi_{i}$ (and also the symbol $W_{i} W_{l i}$ used in $\S 14$ ) is seriously limited by the fact that the expressions cannot be factored into other expressions which by themselves repre-

* Knoblauch, p. 96 ; Forsyth, The Fundamental Magnitudes in the General Theory of Surfaces, Messenger of Mathematics, vol. 32 (1902), no. 5 .

Trans. Am. Math. Soc. 4 
sent the symbol $\pi_{i}$. This it will be remembered was the case in $\S 2$ where the relation $f_{i}^{\prime}=\left(f F^{\prime}\right) F_{i}$ was proved.

$\S 13$. In connection with the coefficients of these forms, it is interesting to notice the differential equation of the lines of curvature through an umbilicus when expressed in the cubic symbols.

This equation is

$$
\begin{aligned}
\psi^{2}[(E Q-F P) \psi+E R-F Q]+\psi[ & (E R-G P) \psi+E S-G Q] \\
& +(F R-G Q) \psi+F S-G R=0 . *
\end{aligned}
$$

The notations here used are explained as follows :

$$
\begin{gathered}
\Psi=\frac{d u_{1}}{d u_{2}}=-\frac{W_{2}}{W_{1}}, \quad E=f_{1}^{2}, \quad F=f_{1} f_{2}, \quad G=f_{2}^{2} . \\
d s^{3} \frac{d r^{-1}}{d s}=P d u_{1}^{3}+3 Q d u_{1}^{2} d u_{2}+3 R d u_{1} d u_{2}^{2}+S d u_{2}^{3} .
\end{gathered}
$$

Therefore $P=\sigma \pi_{i}^{3}, Q=\sigma \pi_{1}^{2} \pi_{2}, R=\sigma \pi_{1} \pi_{2}^{2}, S=\sigma \pi_{2}^{3}$, where $\sigma$ is a factor of proportionality. Then

$$
\begin{array}{ll}
E Q-F P=\sigma f_{1} \pi_{1}^{2}(f \pi), & E S-G Q=\sigma \pi_{2}(f \pi)\left(f_{1} \pi_{2}+f_{2} \pi_{1}\right), \\
E R-F Q=\sigma f_{1} \pi_{1} \pi_{2}(f \pi), & F R-G Q=\sigma f_{2} \pi_{1} \pi_{2}(f \pi), \\
E R-G P=\sigma \pi_{1}(f \pi)\left(f_{1} \pi_{2}+f_{2} \pi_{1}\right), & F S-G R=\sigma f_{2} \pi_{2}^{2}(f \pi) .
\end{array}
$$

The introduction of these values into the differential equation gives the very simple form

$$
(f \pi)(f W)(\pi W)^{2}=0 .
$$

The only complicated part of this function is the symbol $\pi$, but this is no more so than the coefficients $P, Q, R, S$ ordinarily used, each of which must have its separate explanation in terms of other coefficients.

\section{§ 14. Weingakten's formulas fOR the cö̈rdinates of a sURfaCe. $\dagger$}

Let $W$ be the distance from the origin to the tangent plane. Then

$$
W=\Sigma X x, \quad W_{1}=\Sigma X_{1} x, \quad W_{2}=\Sigma X_{2} x .
$$

The equations $\Sigma X X_{1}=0$ and $\Sigma X X_{2}$ give $X, Y, Z$ except for a factor of proportionality. The identity $\Sigma\{X Y\}^{2}=\Sigma X_{1}^{2} \cdot \Sigma X_{2}^{2}-\left(\Sigma X_{1} X_{2}\right)^{2}$ with the definitions of the symbol $f^{\prime}$ shows this factor to be $\beta^{\prime}$ and if $\delta$ be defined by the relations $\beta^{\prime}=\beta \delta$, then $X=\beta^{\prime}\{Y Z\}=\delta(Y Z)$, etc. This relation shows that the determinant of the above system is $\beta^{\prime-1}$ and the solution of the system is

\footnotetext{
* Forsyth, ibid., p. 77.

† BIANCHI, \& 72.
} 


$$
\begin{aligned}
x=\delta(Y Z) & W+\delta(Z W) X+\delta(W Y) Z \\
& =W X+\delta^{2}(Z X)(Z W)+\delta^{2}(Y X)(Y W)+\delta^{2}(X X)(X W) ;
\end{aligned}
$$

the last term being zero is arbitrarily added. By the definition of $f^{\prime}$,

$$
\Sigma(X U)(X W)=\left(f^{\prime} U\right)\left(f^{\prime} W\right)
$$

and therefore $x=W X+\delta^{2}\left(f^{\prime} W\right)\left(f^{\prime} X\right)$, and similarly for $y$ and $z$. It is desired to find an expression for $F_{i} F_{j}$. By definition $-F_{i} F_{j}=\Sigma X_{j} x_{i}$. In order to find this sum, note that

$$
x_{i}=W_{i} X+W X_{i}+\delta\left(f^{\prime} X\right)\left[\delta\left(f^{\prime} W\right)\right]_{i}+\delta\left(f^{\prime} W\right)\left[\delta\left(f^{\prime} X\right)\right]_{i},
$$

and in the reduction of this make use of the relations

$$
\Sigma X_{j}\left(f^{\prime} X\right)=\left(f^{\prime} \phi^{\prime}\right) \phi_{j}^{\prime}, \quad \Sigma X_{j}[\delta(f X)]_{i}=\left[\Sigma \delta X_{j}(f X)\right]_{i}-\delta \Sigma(f X) X_{i j} .
$$

The application of (14) to the case of the sphere gives

whence follows

$$
X_{i j}=F_{i}^{\prime} F_{j}^{\prime} X-\delta^{2}\left(\phi^{\prime} \psi^{\prime}\right)\left(\psi^{\prime} X\right) \phi_{i j}^{\prime},
$$

$$
\Sigma X_{j}[\delta(f X)]_{i}=\left[\delta\left(f^{\prime} \phi^{\prime}\right) \phi_{j}^{\prime}\right]_{i}+\delta^{3}\left(\psi^{\prime} \chi^{\prime}\right)\left(\psi^{\prime} \phi^{\prime}\right)\left(\chi^{\prime} f^{\prime \prime}\right) \phi_{i j}^{\prime} .
$$

By (9) the second term is $\delta\left(\phi^{\prime} f^{\prime}\right) \phi_{i j}^{\prime}$, whence follows

$$
-F_{i} F_{j}=W f_{i}^{\prime} f_{i}^{\prime}+\delta\left(f^{\prime} \phi^{\prime}\right) \phi_{j}^{\prime}\left[\delta\left(f^{\prime} W\right)\right]_{i}+\delta\left(f^{\prime} W\right)\left[\delta\left(f^{\prime} \phi^{\prime}\right)\right]_{i} \phi_{j}^{\prime} .
$$

Consider now this last term for the two cases $j=1,2$. For example, if $j=1$ the coefficient of $W_{2}$ is zero by rule 2 and the coefficient of $W_{1}$ is zero as follows. Since $\delta^{2}\left(f^{\prime} \phi^{\prime}\right)^{2}=2$, therefore

or

$$
\delta\left(f^{\prime} \phi^{\prime}\right)\left[\delta\left(f^{\prime} \phi^{\prime}\right)\right]_{i}=0
$$

whence

$$
\delta f_{1}^{\prime} \phi_{2}\left[\delta\left(f^{\prime} \phi^{\prime}\right)\right]_{i}=\delta f_{2}^{\prime} \phi_{1}^{\prime}\left[\delta\left(f^{\prime} \phi^{\prime}\right)\right]_{i}=-\delta \phi_{2}^{\prime} f_{1}^{\prime}\left[\delta\left(f^{\prime} \phi^{\prime}\right)\right]_{i} \text {, }
$$

$$
\delta f_{1}^{\prime} \phi_{2}^{\prime}\left[\delta\left(f^{\prime} \phi^{\prime}\right)\right]_{i}=0 \text {. }
$$

The final form of the identity is then

$$
-F_{i} F_{j}=W f_{i}^{\prime} f_{j}^{\prime}+\delta\left(f^{\prime} \phi^{\prime}\right) \phi_{j}^{\prime}\left[\delta\left(f^{\prime} W\right)\right]_{i} .
$$

Everywhere, except perhaps in the last term, $i$ and $j$ may be interchanged. Therefore it can be done in this term and consequently the following notation,

$$
\bar{W}_{i} W_{j}=\delta\left(f^{\prime} \phi^{\prime}\right) \phi_{j}^{\prime}\left[\delta\left(f^{\prime} W\right)\right]_{i},
$$

is legitimate. This term $W_{i} W_{j}$ is the function which BranchI uses as the coefficient in the second covariant differential.* The desired result is therefore

$$
-F_{i} F_{i}=f_{i}^{\prime} f_{j}^{\prime} W+\bar{W}_{i} \bar{W}_{j} \text {. }
$$

* This may be seen by comparing the coefficients of the form as given by $\mathrm{BIANCHI}$ in 826 and those of the symbolic form given on p. 203, MASCHKE I. 


\section{§15. The characteristic function.*}

In the problem of deformation of surfaces use is made of an auxiliary function $Q$, defined by the equation $2 Q=\Sigma(x \bar{x})$, where the point $\bar{x}, \bar{y}, \bar{z}$ corresponds to the point $x, y, z$ by orthogonality of elements. The following equations are fundamental :

$$
\Sigma x_{1} \bar{x}_{1}=0, \quad \Sigma x_{2} \bar{x}_{2}=0, \quad \Sigma x_{1} \bar{x}_{2}+\Sigma x_{2} \bar{x}_{1}=0 .
$$

The last of these with the equation for $Q$ gives the two equations

$$
\beta \Sigma x_{1} \bar{x}_{2}=-\beta \Sigma x_{2} \bar{x}_{1}=Q \text {. }
$$

From the equation for $Q$ it follows by differentiation that

$$
2 Q_{\lambda}=\Sigma(x \bar{x})_{\lambda}=\Sigma\left(x_{\lambda} \bar{x}\right)+\Sigma\left(x \bar{x}_{\lambda}\right)+\beta_{\lambda} \Sigma\{x \bar{x}\} .
$$

From this the terms $\bar{x}_{\lambda i}$ are removed as follows:

$$
\Sigma\left(x \bar{x}_{\lambda}\right)=\beta \Sigma x_{1} \bar{x}_{\lambda 2}-\beta \Sigma x_{2} \bar{x}_{\lambda 1} .
$$

From the fundamental equations,

whence

$$
\Sigma x_{1} \bar{x}_{\lambda 2}=-\Sigma x_{\lambda 2} \bar{x}_{1}, \quad \Sigma x_{2} \bar{x}_{\lambda 1}=-\Sigma x_{\lambda 1} \bar{x}_{2},
$$

This gives

$$
\Sigma\left(x \bar{x}_{\lambda}\right)=\Sigma\left(x_{\lambda} \bar{x}\right) \text {. }
$$

From (14) follows

$$
2 Q_{\lambda}=2 \Sigma\left(x_{\lambda} \bar{x}\right)+\beta_{\lambda} \Sigma\{x \bar{x}\} \text {. }
$$

$$
\Sigma\left(x_{\lambda} \bar{x}\right)=F_{\lambda} \Sigma X\left(F^{\prime} \bar{x}\right)-(f \phi) \Sigma(\phi x)\left(f_{\lambda} \bar{x}\right) .
$$

The last term must be expanded and in the expansion

$$
\Sigma x_{1} \bar{x}_{1}=\Sigma x_{2} \bar{x}_{2}=0 \quad \text { and } \quad \Sigma x_{1} \bar{x}_{2}=-\Sigma x_{2} \bar{x}_{1} .
$$

The term then becomes

$$
\beta(f \phi)\left\{\phi f_{\lambda}\right\} \Sigma x_{2} \bar{x}_{1} .
$$

By interchange of $f$ and $\phi$ and by the relation $Q=-\beta \Sigma x_{2} \bar{x}_{1}$, this is reduced to $-\frac{1}{2} Q \beta^{2}\left[\{f \phi\}^{2}\right]_{\lambda}$, which by $(8)$ is $Q \beta^{-1} \beta_{\lambda}$. The equation for $Q$ then becomes

From (16) it follows that

$$
\beta Q_{\lambda}=F_{\lambda} \Sigma X(F \bar{x})
$$

This may also be written

$$
F_{\lambda}(F Q)=-K \Sigma X \bar{x}_{\lambda}
$$

and from this is obtained

$$
\Sigma X \bar{x}_{\lambda}=-\delta(F Q) F_{\lambda},
$$

$$
\Sigma(X \bar{x})=(F, \delta(F Q)) \text {. }
$$

\footnotetext{
* BianchI, \& 154.
} 
The left member is evaluated by removing $X_{i}$ by (15) and the summations by the fundamental equations. The result is

$$
(F, \delta(F Q))=-(f F)^{2} Q
$$

It is now desirable to express this relation without the use of the expressions $F_{i} F_{j k}$ and by means of the symbols $f^{\prime}$ instead of $f$. The change is made as follows :

$$
\begin{aligned}
(F, \delta(F Q)) & =\delta(F,(F Q))+(F Q)(F \delta), \\
(F,(F Q)) & =-\overline{F F Q}+\overline{F Q F}+(F Q)\{F \beta\},
\end{aligned}
$$

and by (6)

$$
\overline{F Q F}=\overline{Q F F} .
$$

The expression $\overline{Q F F}$ is simplified by the Codazzi formulas, viz.,

$$
\left(F F_{\lambda}\right)=-(f \phi)(F \phi)\left(F f_{\lambda}\right) .
$$

The terms containing $\delta F_{k}\left(F Q_{j}\right)$ are changed as follows :

$$
\begin{aligned}
\delta F_{k}\left(F Q_{j}\right) & =\frac{1}{2} \delta^{3}\left(f^{\prime} \phi^{\prime}\right)^{2}\left(F Q_{j}\right) F_{k} \text { by }(8) \\
& =\delta^{3}\left(f^{\prime} \phi^{\prime}\right)\left(f^{\prime} F\right)\left(\phi^{\prime} Q_{j}\right) F_{k}
\end{aligned}
$$

by means of (4) applied to $\left(f^{\prime} \phi^{\prime}\right)\left(F Q_{j}\right)$ and then rule 1.

The symbols $f$ and $\phi$ occur in the terms $(f \phi)(F \phi)\left(F f_{k}\right)$ introduced by the Codazzi formulas. They may be removed by the relations $f_{i}=-\delta\left(f^{\prime} \Phi\right) \Phi_{i}$, $\phi_{i}=-\delta\left(\phi^{\prime} \Psi\right) \Psi_{i}$. These lead to the relations $(f \phi)=\delta\left(f^{\prime} \phi^{\prime}\right)$ by rule 1 and (16), $(F \phi)=-\delta\left(\phi^{\prime} \Psi\right)\left(F^{\prime} \Psi\right)$, and $\left(F f_{k}\right)=-\left[\delta\left(f^{\prime} \Phi\right)\right]_{k}(F \Phi)-\delta\left(f^{\prime} \Phi\right)\left(F \Phi_{k}\right)$. As a result of these changes there is introduced a term

$-\delta^{3}\left(f^{\prime} \phi^{\prime}\right)\left(\phi^{\prime} \Psi\right)\left(F^{\prime} \Psi\right)\left[-\beta Q_{2}\left[\delta\left(f^{\prime} \Phi\right)\right]_{1}(F \Phi)\right.$

$$
\left.+\beta Q_{1}\left[\delta\left(f^{\prime} \Phi\right)\right]_{2}(F \Phi)-\beta Q_{2} \delta\left(f^{\prime} \Phi\right)\left(F \Phi_{1}\right)+\beta Q_{1}\left(f^{\prime} \Phi\right)\left(F \Phi_{2}\right)\right] \text {. }
$$

To the expressions $\left(\phi^{\prime} \Psi\right)(F \Phi)$ and $\left(\phi^{\prime} \Psi\right)\left(F \Phi_{k}\right)$ which occur in this term apply (4) and use the relation $\delta\left(F^{\prime} \Psi\right)^{2}=2$. These reductions lead to

$$
\begin{aligned}
& (F, \delta(F Q))=\delta^{3}\left(f^{\prime} \phi^{\prime}\right)\left(f^{\prime} F^{\prime}\right)\left[\beta F_{1}\left(\phi^{\prime} Q_{2}\right)-\beta F_{2}\left(\phi^{\prime} Q_{1}\right)\right] \\
& +\delta(F Q)\{F \beta\}-\delta^{2}\left(f^{\prime} \phi^{\prime}\right)\left(\phi^{\prime} \Phi\right)\left[-\beta Q_{2}\left[\delta\left(f^{\prime} \Phi\right)\right]_{1}+\beta Q_{1}\left[\delta\left(f^{\prime} \Phi\right)\right]_{2}\right] \\
& \delta^{2}\left(f^{\prime} \phi^{\prime}\right)\left(f^{\prime} \Phi\right)\left[-\beta Q_{2} \delta\left(\phi^{\prime} \Phi_{1}\right)+\beta Q_{1} \delta\left(\phi^{\prime} \Phi_{2}\right)\right]+(F Q)(F \delta) .
\end{aligned}
$$

If $\delta(F Q)\{F \beta\}$ and $(F Q)\{F /\}$ be multiplied by $\frac{1}{2} \delta^{2}\left(f^{\prime} \phi^{\prime}\right)^{2}$, they can be written (by (4) and rule 1) in the forms $\delta^{3}\left(f^{\prime} \phi^{\prime}\right)\left(f^{\prime} F^{\prime}\right)\left(\phi^{\prime} Q\right)\{F \beta\}$ and $\delta^{2}\left(f^{\prime} \phi^{\prime}\right)\left(f^{\prime} F^{\prime}\right)\left(\phi^{\prime} Q\right)(F \delta)$ respectively.

In the third and fourth terms $\Phi$ is to be replaced by $F$, and in the third $f^{\prime}$ and $\phi^{\prime}$ interchanged. If now for $\left[\delta\left(\phi^{\prime} F^{\prime}\right)\right]_{k}$ one substitutes 


$$
\delta_{k}\left(\phi^{\prime} F^{\prime}\right)+\delta\left(\phi_{k}^{\prime} F\right)+\delta\left(\phi^{\prime} F_{k}\right)+\delta\left\{\phi^{\prime} F^{\prime}\right\} \beta_{k}
$$

and groups the terms according to $(7)$, the result is the desired relation. In this final reduction the coefficients of $(Q \delta)$ and $(Q \beta)$ are zero. Therefore

$$
\delta(F ; \delta(F Q))=\delta^{3}\left(f^{\prime} \phi^{\prime}\right)\left(f^{\prime} F\right)\left(F,\left(\phi^{\prime} Q\right)\right) .
$$

The expression $\left(f F^{\prime}\right)^{2}$ when transformed by the elimination of $f$ becomes $\delta\left(f^{\prime} F^{\prime}\right)^{2}$ and the characteristic equation is accordingly

$$
\delta^{3}\left(f^{\prime} \phi^{\prime}\right)\left(f^{\prime} F\right)\left(F, \delta\left(\phi^{\prime} Q\right)\right)=-\delta^{2}\left(f^{\prime} F^{\prime}\right)^{2} Q .
$$

Into this equation the symbols $\bar{Q}_{i} \bar{Q}_{j}$ are easily introduced. By definition $\bar{Q}_{i} \bar{Q}_{j}=-\delta\left(f^{\prime} \phi^{\prime}\right) f_{i}^{\prime}\left[\delta\left(\phi^{\prime} Q\right)\right]_{j}$ and the equation may therefore be written

$$
(F \bar{Q})^{2}=-\left(f^{\prime} F\right)^{2} Q
$$

It has been proved that if $W$ be the distance from the origin to the tangent plane, $-F_{i} F_{j}=f_{i}^{\prime} f_{j}^{\prime} W+\bar{W}_{i} \bar{W}_{j}$. If now the symbols $F^{\prime}$ be removed by this relation, the characteristic equation becomes

$$
\left(f^{\prime} \bar{Q}\right)^{2} W+\left(f^{\prime} \bar{W}\right)^{2} Q=\left(f^{\prime} \phi^{\prime}\right)^{2} W Q-(\bar{W} \bar{Q})^{2} .
$$

It is to be noted that the functions $W$ and $Q$ enter into this equation symmetrically and from this follows the theorem :*

If $S_{0}$ denote the envelope of the planes $\Sigma X x=W$ and if $Q$ be the characteristic function for an infinitely small deformation of the surface $S$, then is $W$ the characteristic function for an infinitely small deformation of the surface $S_{0}$.

\section{§ 16. Rectilinear congruences.}

The analytical definition of rectilinear congruences is as follows: The entire system of rays is cut by a surface $S$ and that point (or one of them if there are more than one) in which the ray cuts the surface is taken as the origin of each ray. The surface $S$ is defined analytically by a curvilinear system of coördinates $\left(u_{1}, u_{2}\right)$ and any ray is determined from its origin $x, y, z$ and its direction cosines $X, Y, Z$, all expressed as functions of $u_{1}$ and $u_{2}$. The spherical image of any ray is the point $X, Y, Z$ on the sphere. Only in special cases are the rays perpendicular to $S$.

The forms $d \sigma^{2}=\Sigma d X^{2}$ and $\Sigma d x d X$ are fundamental. The first of these is the Gaussian arc form, but since the first fundamental form is not here considered, the accents on the symbols for $d \sigma^{2}$ and on $\beta^{\prime}$ will be omitted. Whenever these are introduced again, every ( ) must be replaced by $\delta()$. The symbols used are $f_{i} f_{j}=\Sigma X_{i} X_{j}$ and $F_{i} \overline{F_{j}}=\Sigma X_{i} x_{j}$. It is to be noticed that the symbols $F_{i}$ and $\bar{F}_{i}$ are not interchangeable in the general case and that it takes a product of the two for the actual meaning.

\footnotetext{
* BIANCHI, § 155 .
} 
Let now $\cos \lambda, \cos \mu, \cos \nu$ be the direction cosines and $d l$ the length of the common perpendicular from $\left(u_{1} u_{2}\right)$ to the consecutive ray $\left(u_{1}+d u_{1}, u_{2}+d u_{2}\right)$. The direction cosines of the two rays are $X, Y, Z$ and $X+d X, Y+d Y$, $Z+d Z$. Then $\Sigma X \cos \lambda=0$ and $\Sigma(X+d X) \cos \lambda=0$ and therefore

$\cos \lambda: \cos \mu: \cos \nu=(Y d Z-Z d Y):(Z d X-X d Z):(X d Y-Y d X)$.

From the equations $\Sigma X X_{i}=0$ follows $X=(Y Z)$. Let $W$ be a curve on $S$, and $p$ be defined as before. Then from the notations in use

$$
Y d Z-Z d Y=(f X)\left\lceil f_{1} d u_{1}+f_{2} d u_{2}\right]=p(f X)(f W) .
$$

Therefore $\cos \lambda: \cos \mu: \cos \nu=(. f X)(f W):(f Y)(f W):(f Z)\left(f^{\prime} W\right)$ and the solution of this is $\cos \lambda=p^{2}(f X)(f W)$, etc.

The length of the line between the two rays is

$$
d l=\Sigma \cos \lambda d x=p^{3}\left(f F^{\prime}\right)(f W)(\bar{F} W) .
$$

If now $r$ be the distance along a ray from its origin to its intersection with $d l$, then it may be proved that

$$
r=-\frac{\Sigma d X d x}{\Sigma d X^{2}}=-p^{2}(F W)(\bar{F} W) \cdot *
$$

\section{§ 17. Principal curves.}

Introduce now conditions which in form of expression are similar to those imposed for lines of curvature. Let $U$ and $V$ be curves which satisfy the equations $(f U)(f V)=0$ and $(F U)(\bar{F} V)+(\bar{F} U)(F V)=0$. These are the principal curves. Let $r_{1}$ and $r_{2}$ be the values of $r$ for rays whose origins are on these curves. Then $r_{1}=-p^{2}(F U)(\bar{F} U)$ and $r_{2}=-q^{2}(F V)(\bar{F} V)$. The first of the conditions on $U$ and $V$ may be written $V_{i}=m(f U) f_{i}$ and by substitution of this it follows that

$$
r_{2}=-p^{2}(f F)(\phi \bar{F})(f U)(\phi U) .
$$

Consider now three rays, one the original ray at the intersection of $U$ and $V$, the two others near to this, one on $U$ and one on $W$, a curve copunctual with $U$ and $V$. From the original ray to each of the others there is one line which is perpendicular to both. Let $\omega$ be the angle between these two lines. Then by the formulas proved above (the subscripts referring to the curves)

$$
\cos \omega=\Sigma \cos \lambda_{W} \cos \lambda_{U}=\frac{p(f W)(\phi U) \Sigma(f X)(\phi X)}{1^{\prime}\left(f^{\prime} W\right)^{2}}=\frac{p(f W)(f U)}{\sqrt{(f W)^{2}}},
$$

by (17) and (9). From this follows, as in $\S 2$,

$$
\cos ^{2} \omega=\frac{p^{2}(f W)(\phi W)(f U)(\phi U)}{(f \bar{W})^{2}} \quad \text { and } \quad \sin ^{2} \omega=\frac{p^{2}(U W)^{2}}{(f W)^{2}}
$$

\footnotetext{
* BIA NCHI, \& 137.
} 
Form now the expression $-\left(r_{1} \cos ^{2} \omega+r_{2} \sin ^{2} \omega\right)$ as a function of $U$ and $W$. Except for the difference in notations and the conditional equations, this problem is that of Euler's formula and may be proved by exactly the same method as was employed in the former problem ( $\$ 5)$. The result is Hamilton's equation, $r=r_{1} \cos ^{2} \omega+r_{2} \sin ^{2} \omega$, where $r$ has the value pertaining to the curve $W$.

The maximum and minimum properties of $r_{1}$ and $r_{2}$ may be proved. Suppose $r_{2}>r_{1}$. Then from Hamilton's equation

$$
\begin{aligned}
& r-r_{1}=\left(r_{2}-r_{1}\right) \sin ^{2} \omega, \\
& r-r_{2}=\left(r_{1}-r_{2}\right) \cos ^{2} \omega
\end{aligned}
$$

and therefore $r_{1}<r<r_{2}$. The intersection $L_{1}$ of the ray $\left(u_{1}, u_{2}\right)$ and the common perpendicular between this ray and the consecutive ray on $U$ is called a limit point. A similar point, $L_{2}$, exists for the curve $V$. The angle between these two perpendiculars is obtained from the formula for $\cos \omega$ if $W$ is replaced by $V$. The numerator in that case has the factor $(f U)(f V)$. Therefore the lines are orthogonal.

In the exceptional case where the coefficients of the two forms are proportional, the notations give $F_{j}=k f_{j}, \bar{F}_{j}-k f_{j}$ and therefore $r=r_{1}=r_{2}=-k \bar{k}$.

Any curve $W\left(u_{1}, u_{2}\right)=0$ on $S$ determines a system of rays which form a ruled surface. Principal suifaces will be those determined in this manner by the principal curves $U$ and $V$. The differential equation of the principal surfaces is found from the conditions on $U$ and $V$ and is

$$
\left[\left(F^{\prime} W\right)(f \bar{F})+(\bar{F} W)(f F)\right](f W)=0 .
$$

\section{$\S 18$. The QUADRaTIC IN $r$.}

Owing to the differences in notation the derivation of this equation cannot be referred back to the work on the similar problem in the case of the radii of curvature.

Consider first $-\left(r_{1}+r_{2}\right)$. This may be reduced by repeated applications of (4) to $p^{2} q^{2}\left(f F^{\prime}\right)\left(f \overline{F^{\prime}}\right)(V U)^{2}$. But since for $U$ and $V \sin ^{2} \omega=1$, this becomes $\left(f F^{\prime}\right)(f \bar{F})$.

Also $r_{1} r_{2}=p^{2} q^{2}(F V)(\bar{F} V)(\Phi U)(\bar{\Phi} U)$. Aside from the factor $p^{2} q^{2}$ this is

or

$$
(F V)(\Phi U)[(\bar{F} \bar{\Phi})(V U)+(\bar{F} U)(\bar{\Phi} V)]
$$

$$
(F V)(\bar{\Phi} U)[(\bar{F} \Phi)(V U)+(\bar{F} U) \dot{(} \Phi V)] \text {. }
$$

From the addition of these two expressions together with the second condition on $U$ and $V$ follows

$$
2 r_{1} r_{2}=p^{2} q^{2}(\bar{F} \bar{\Phi})(F V)(\Phi U)(V U)+p^{2} q^{2}(\bar{F} \Phi)(F V)(\bar{\Phi} U)(V U) \text {. }
$$


The pairs of symbols $F, \bar{F}$ and $\Phi, \Phi$ may always be interchanged and therefore $2 p^{2} q^{2}(\bar{F} \bar{\Phi})(F V)(\Phi U)(V U)=p^{2} q^{2}(F \Phi)(\bar{F} \bar{\Phi})(V U)^{2}=(F \Phi)(\bar{F} \bar{\Phi})$. By (4),

$p^{2} q^{2}(\bar{F} \Phi)(F V)(\Phi U)(V U)=(\bar{F} \Phi)(F \bar{\Phi})-p^{2} q^{2}(\bar{F} \Phi)(F U)(\bar{\Phi} V)(V U)$.

From these results may be written

$$
4 r_{1} r_{2}=(F \Phi)(\bar{F} \bar{\Phi})+2(F \Phi)(\bar{F} \Phi)-2 p^{2} q^{2}(\bar{F} \Phi)(F U)(\Phi V)(V U) \text {. }
$$

In every term, except perhaps the last, $F$ and $\bar{F}, \Phi$ and $\Phi$ may be simultaneously interchanged, and therefore also in the last. Thus

$$
\begin{aligned}
-2 p^{2} q^{2}(\bar{F} \Phi)(F U) & (\Phi V)(V U)=-2 p^{2} q^{2}(F \bar{\Phi})(\bar{F} U)(\Phi V)(V U) \\
& =-2 p^{2} q^{2}(\Phi \bar{F})(\bar{\Phi} U)(F V)(V U)=-(F \bar{\Phi})(\bar{F} \Phi)
\end{aligned}
$$

by the process of (9). The substitution of this result reduces the relation to

$$
4 r_{1} r_{2}=(F \Phi)(\bar{F} \bar{\Phi})+(F \Phi)(\bar{F} \Phi)
$$

and the desired equation is

$$
4 r^{2}+4(f F)(. \bar{F}) r+(F \Phi)(\bar{F} \Phi)+(F \Phi)(\bar{F} \Phi)=0
$$

\section{§ 19. Developable surfaces.}

Developable surfaces are defined as those for which the consecutive rays intersect. If $W$ defines a curve such that the corresponding system of rays form a developable surface, then must $W$ satisfy the differential equation $d l=0$, that is, $W$ must satisfy

$$
\left(f F^{\prime}\right)(f W)(\bar{F} W)=0 .
$$

There are then through each point of $S$ two developable surfaces, real or imaginary. Consider now two curves $W$ and $T$ which are defined by the relations $(f W)(f T)=0$ and $(F W)(\bar{F} T)=0[$ or $(\bar{F} W)(F T)=0]$. These two conditions are distinct from those for principal curves, the second condition in this case requiring more than the corresponding condition for principal curves. From the first of these conditions follows $W_{i}=n(f T) f_{i}$ or $T_{i}=m(f W) f_{i}$. The substitution of either of these relations into the second condition shows that the surfaces defined by these curves are developable surfaces.

Let the ray through a point $P$ meet the cuspidal edges of these surfaces in the points $P_{1}$ and $P_{2}$, which are called foci of the system. Let also $\rho_{1}$ and $\rho_{2}$ be the distances $P P_{1}$ and $P P_{2}$ respectively. If by $\xi, \eta, \zeta$ are denoted the coördinates of the cuspidal edge, then $\xi=x+\rho X$, etc., and since the ray is tangent to the cuspidal edge at the focus, it follows that $d x+\rho d X=\lambda X$, 
where $\lambda$ is a factor of proportionality. This last relation may be written $(x W)+\rho(X W)=\lambda X$ and therefore for $j=1,2, \Sigma X_{j}(x W)+\rho \Sigma X_{j}(X W)=0$. This equation expressed in symbols is $(\bar{F} W) F_{j}+\rho(f W) f_{j}=0$. Let the value $\rho_{1}$ go with $W$ and $\rho_{2}$ with $T$. These give rise to the following system of equations :

1) $F_{1}(\bar{F} W)+\rho_{1} f_{1}(f W)=0$,

3) $\Phi_{1}(\bar{\Phi} T)+\rho_{2} \phi_{1}(\phi T)=0$,

2) $F_{2}(\bar{F} W)+\rho_{1} f_{2}(f W)=0$,

4) $\Phi_{2}(\bar{\Phi} T)+\rho_{2} \phi_{2}(\phi T)=0$.

From the multiplication of 1) by 4) and 2) by 3) follow

$$
\begin{aligned}
& F_{1} \Phi_{2}\left(\overline{F^{\prime}} W\right)(\bar{\Phi} T)=\rho_{1} \rho_{2} f_{1} \phi_{2}(f W)(\phi T), \\
& F_{2} \Phi_{1}(\bar{F} W)(\bar{\Phi} T)=\rho_{1} \rho_{2} f_{2} \phi_{1}(f W)(\phi T) .
\end{aligned}
$$

The subtraction of these two equations and the interchange of equivalent symbols gives

$$
2 \rho_{1} \rho_{2}=(F \Phi)(\bar{F} \bar{\Phi}) \text {. }
$$

Now multiply equation 1) by $(\phi T) \phi_{2}$ and 2) by $(\phi T) \phi_{1}$, subtract them, and then do the same for 3 ) and 4). With proper changes of notation this gives the two equations:

$$
\begin{aligned}
& (F \phi)(\phi T)(\bar{F} W)+\rho_{1}(f \phi)(\phi T)(f W)=0, \\
& (F \phi)(\phi W)(\bar{F} T)-\rho_{2}(f \phi)(\phi T)(f W)=0 .
\end{aligned}
$$

From these equations is obtained $\rho_{1}+\rho_{2}=-(f F)(f \bar{F})$, and therefore the desired quadratic is

$$
2 \rho^{2}+2\left(f F^{\prime}\right)(f \bar{F}) \rho+(F \Phi)(\bar{F} \bar{\Phi})=0 .
$$

It is at once evident that $r_{1}+r_{2}=\rho_{1}+\rho_{2}$.

The point midway between the foci, and so midway between the principal points also, is called the mid-point and its locus is the mid-surface.

The four equations $(A),(B),(C),(D)$ are here given with the complete notation. In the next section the full form is needed.

$$
\delta^{3}\left[\left(F^{\prime} W\right)\left(f^{\prime} \overline{F^{\prime}}\right)+\left(\overline{F^{\prime}} W\right)\left(f^{\prime} F^{\prime}\right)\right]\left(f^{\prime \prime} W\right)=0,
$$$$
\text { (B) } 4 r^{2}+4 \delta^{2}\left(f^{\prime} F\right)\left(f^{\prime} \bar{F}\right) r+\delta^{2}(F \Phi)(\bar{F} \bar{\Phi})+\delta^{2}(F \bar{\Phi})(\bar{F} \Phi)=0 \text {, }
$$

$$
\begin{aligned}
\delta^{3}\left(f^{\prime} F\right)\left(f^{\prime} W\right)(\bar{F} W) & =0, \\
2 \rho^{2}+2 \delta^{2}\left(f^{\prime} F\right)\left(f^{\prime} \bar{F}\right) \rho+\delta^{2}(F \Phi)(\bar{F} \bar{\Phi}) & =0 .
\end{aligned}
$$




\section{§20. The coincidence of the focal surfaces.*}

It may be shown that if the two focal surfaces coincide the cuspidal edges unite into a system of asymptotic lines. Assume this to be the case and choose for $S$ the combined focal surfaces. Since now the ordinary surface elements enter into the work, the symbols $f$ and $f^{\prime}$ are to be distinguished. Let $U$ be the system of asymptotic lines, $V$ their orthogonal trajectories. Then $\left(F C^{T}\right)^{2}=0$. Furthermore let $X^{\prime}, Y^{\prime}, Z^{\prime}$ be the direction cosines of the tangent to the curve $U$; $X^{\prime \prime}, Y^{\prime \prime}, Z^{\prime \prime}$ of the tangent to $V$; and $X, Y, Z$ of the normal to $S$. Then from $\$ 11$,

where

$$
X_{\lambda}^{\prime}=R_{\lambda} X+T_{\lambda} X^{\prime \prime}
$$

$$
\begin{aligned}
X^{\prime} & =p(x U), & X^{\prime \prime} & =q(x V), \text { etc. }, \\
R_{\lambda} & =p(F U) F_{\lambda}, & T_{\lambda} & =m t(f \phi)(\phi U)(f U)_{\lambda} .
\end{aligned}
$$

These equations involve only the orthogonality of $U$ and $V$. According to the hypothesis $X^{\prime}, Y^{\prime}, Z^{\prime}$ are the direction cosines of the rays. Expressions are next to be found for the rectilinear congruence symbols. In this computation it is to be noted that $R_{\lambda}$ and $T_{\lambda}$ have actual meaning and are not symbols in the technical sense.

From the relations $\Sigma X^{2}=\Sigma X^{\prime 2}=\Sigma X^{\prime \prime 2}=1, \Sigma X^{\prime \prime} X=\Sigma X x_{j}=0$, $\Sigma X^{\prime \prime} x_{j}=q \Sigma(x V) x_{j}=q(f V) f_{j}$ it follows that

$$
f_{i}^{\prime} f_{j}^{\prime}=\Sigma X_{i}^{\prime} X_{j}^{\prime}=R_{i} R_{j}+T_{i} T_{j}, \quad F_{i} \bar{F}_{j}=\Sigma X_{i}^{\prime} x_{j}=q(f V) T_{i} f_{j} .
$$

The next step is the investigation of the quadratic for $r$, viz :

$$
4 r^{2}+4 \delta^{2}\left(f^{\prime} F\right)\left(f^{\prime} \bar{F}\right) r+\delta^{2}(F \Phi)(\bar{F} \bar{\Phi})+\delta^{2}(F \bar{\Phi})(\bar{F} \Phi)=0 .
$$

Consider now the coefficient of $r$, which may be written

$$
4 \delta^{2} p q m t(f F)(f V)(F U)(\phi \psi)(\psi U)((\phi U), R) .
$$

(In the reduction to this form remove $F_{i} \bar{F}_{j}$ first, then $f_{i}^{\prime} f_{j}^{\prime}$.)

Since $(f F)(\psi U)=(\psi F)(f U)+(F U)(f \psi)$, the above expression is zero on account of the conditions $(f U)(f V)=0$ and $(F U)^{2}=0$.

The expression $(F \Phi)(\bar{F} \bar{\Phi})+(F \bar{\Phi})(\bar{F} \Phi)=0$ may be written

$$
q^{2}(f V)(\phi V)[(T T)(f \phi)+(T \phi)(f T)] .
$$

The first term is zero and the second is $-q^{2}[(f V)(f T)]^{2}$, which, by the removal of $T$ and the usual reduction of the number of symbols, becomes $-p^{4} q^{2}[(f V)((f U), V)]^{2}$.

Consider now $\beta^{2} \beta^{\prime-2}=(R T)^{2}$, which is easily derived from the notations. In the expression for $(R T)$ apply $(4)$ to $(\phi U)(F,(f U))$. This reduces

\footnotetext{
* Bianchi, \& 142.
} 
$(R T)$ to $p m t(f \phi)(\phi F)(F U)(U,(f U))$, and if this expression be squared and (4) be applied to $(F U)(\chi \Phi)$, the result is $(R T)^{2}=p^{2} m^{2} t^{2}(f \phi)(\psi \chi)(\phi F)(F \Phi)(\chi U)(\Phi U)(U,(f U))(U,(\psi U))$. Apply the method of (9) to $(F \Phi)(\phi F)(\Phi U)$, and in the result put $m(f \phi)(\phi U)=(f V)$ and $m(\psi \chi)(\chi U)=(\psi V)$.

This gives as a final result $\beta^{2} \beta^{\prime-2}=-p^{2} t^{2}(f V)(U,(f U)) K$. If these results are now substituted in the quadratic for $r$, and reductions made by use of the relations $m q=p, m t=p^{2}$, and $\beta \delta=\beta^{\prime}$, it follows that $4 r^{2}=-K$. Whence the theorem:

If the two focal surfaces coincide, then $4 r^{2}=-K$, or in other words, the distance between the principal points is $1 / \sqrt{-K}$.

HamiLton, N. Y. 